\title{
Consideraciones políticas de la asignación presupuestal en salud en México 2010-2017
}

\section{Political factors in health budget allocation in Mexico 2010-2017}

\author{
Gabriela Tapia Téllez \\ Universidad Autónoma de la Ciudad de México (México) \\ ORCID: https://orcid.org/0000-0002-0929-6130 \\ gabriela.tapia@flacso.edu.mx
}

\begin{abstract}
NOTA BIOGRÁFICA
Doctora en Ciencias Sociales con especialidad en Ciencia Política por FLACSO-México. Maestra en Política Pública y Administración por la Universidad de Nottingham en Reino Unido. Actualmente es Profesora/ Investigadora de la Universidad Autónoma de la Ciudad de México en la licenciatura de Ciencia Política y Administración Urbana y Tutora a distancia de la Maestría en Política Pública Comparada en FLACSOMéxico. Miembro del Sistema Nacional de Investigadores. Sus publicaciones e investigaciones se centran en temas de política pública, política social, presupuesto y transparencia y rendición de cuentas.
\end{abstract}

\section{RESUMEN}

La investigación da seguimiento, desde un lente político/institucional conocido como «the policy making process», al presupuesto en salud para entidades federativas en México en el periodo 2010-2017. El objetivo de la investigación es observar la influencia de variables político/institucionales en la asignación con énfasis en el Órgano Ejecutivo y Legislativo en tres legislaturas (LXI, LXII y LXIII) y dos sexenios (uno con presidencia del Partido Acción Nacional (PAN) y otro con presidencia del Partido Revolucionario Institucional (PRI)). Se observaron dos relaciones: 1. La relación entre el partido político en la presidencia y el partido político del gobernador de cada entidad. 2. La relación entre el partido político con mayoría en la Cámara de Diputados y el partido político del gobernador de cada entidad. Las relaciones se observaron a través del cálculo de medias aritméticas de asignación por sexenio para: 1. Entidades gobernadas por el mismo partido que ostenta la presidencia 2. Todas las entidades federativas y 3 . Entidades gobernadas por la oposición. Encontramos que durante el sexenio del PAN no se observa politización, las entidades gobernadas por la oposición incluso recibieron en promedio más recursos. Con el PRI sin embargo, entre 2013 y 2017 de manera sistemática e ininterrumpida la cantidad promedio de recursos asignados a entidades federativas gobernadas por su partido siempre fue mayor. Además, en todos los años en los que el PRI tuvo mayoría absoluta en la Cámara de Diputados (2010-2017) las entidades gobernadas por el PRI recibieron en promedio más recursos en salud que las entidades gobernadas por la oposición.

\section{PALABRAS CLAVE}

Entidades federativas; México; politización; presupuesto; salud.

\section{ABSTRACT}

Research analyzes, from an institucional/political perspective known as «the policy making process», health budget allocation for localities in México during 2010-2017. The main objective of the research was to observe the influence of political/institutional variables of allocation focusing on the Executive and Legislative branch in two presidential periods and three legislatures. Two types of relations were observed: 1 . The relationship between political party at the presidency and political party at each entity. 2 . The relationship 
between political party with majority in the Lower Chamber and political party governing the entity. The relations were observed through arithmetic averages of allocation for each period for: 1. Localities governed by the same political party than the presidential one 2. All localities 3. Localities governed by opposition. We found that politisation of allocation depends on the political party at the presidency. During the presidency of Partido Acción Nacional (PAN) we do not observe more allocation towards entities governed by PAN, on the contrary, entities governed by opposition received in average more health resources. With Partido Revolucionario Institucional (PRI) however, we observe a clear politisation of health resource allocation. Between 2013-2017 every year there is a coincidence between PRI presidency and resource allocation for localities governed by PRI. Also, every year PRI had majority in the Lower Chamber (2010-2017) entities governed by PRI received in average more health resources than entities governed by opposition.

\section{KEYWORDS}

Budget; health; localities; Mexico; politisation.

\section{SUMARIO}

1. INTRODUCCIÓN. 1.1. PRESUPUESTO Y POLÍTICA. 2. MARCO TEÓRICO: THE POLICY MAKING PROCESS. 2.1. ACTORES EN EL PROCESO DE FORMULACIÓN Y APROBACIÓN DEL PEF EN MÉXICO. 2.1.1. El Ejecutivo. 2.1.2. EI Legislativo. 3. METODOLOGÍA. 3.1. LA VARIABLE PARTIDO DEL PRESIDENTE COMO EXPLICATIVAA LAASIGNACIÓN DE RECURSOS EN SALUD A LAS ENTIDADES FEDERATIVAS. 3.1.1. La No politización de las asignaciones en salud durante el sexenio de Felipe Calderón. 3.1.2. La politización de las asignaciones en el sexenio de Enrique Peña Nieto. 3.2. LA VARIABLE MAYORÍA EN LA CÁMARA DE DIPUTADOS COMO EXPLICATIVA A LA ASIGNACIÓN DE RECURSOS EN SALUD A LAS ENTIDADES FEDERATIVAS. 4. CONCLUSIONES. REFERENCIAS BIBLIOGRÁFICAS.

\section{INTRODUCCIÓN}

Estudiar la manera en que se distribuyen los recursos en salud por entidad federativa es importante por muchas razones. Por un lado, el financiamiento federal de la política social representa para algunos estados en México hasta el 85 por ciento de sus ingresos totales (Medrano y M. Smith, 2017). El presupuesto en salud 2018 representó el $15 \%$ del gasto programable del sector público: 564, 937.5 millones de pesos (Belisario Domínguez, 2018: 1) y el presupuesto para programas y proyectos de inversión durante 2010 a 2017 representó para el IMSS e ISSSTE casi el $40 \%$ de su presupuesto.

La propuesta anual de presupuesto en México tiene origen en el Órgano Ejecutivo y depende de la aprobación del Legislativo, específicamente, de la Cámara de Diputados. El Presupuesto de Egresos de la Federación (PEF), contempla entre muchas otras asignaciones, la cantidad de recursos autorizados para entidades federativas. En la década de los ochenta, la concentración de la asignación de los recursos en salud en el Ejecutivo y Legislativo Federal tenía entre sus principales objetivos disminuir la inequidad entre regiones y mejorar la eficacia del gasto. A pesar de ello, en México la desigualdad en materia de acceso y calidad de los servicios de salud entre entidades cada vez es mayor (CONEVAL, 2018), por lo que los recursos destinados a la construcción de hospitales y equipamiento son primordiales no sólo para la equidad sino en materia de derechos humanos.

La mayor parte de la investigación que existe sobre gasto en salud en México se concentra en la cantidad de recursos que se distribuyen por institución, per cápita y por enfermedad. Son pocas las investigaciones que analizan la efectividad del gasto y contadas las que intentan explicar el peso que tienen distintos factores (políticos, económicos y sociales) en la asignación hacia las entidades federativas. Esto a pesar de la importancia de las coyunturas políticas en las decisiones públicas (Majone, 1989) y que desde hace algunos años México Evalúa (2011) ${ }^{1}$ identificó el uso político del presupuesto en salud como uno de los cuatro elementos que explican la falta de calidad y eficiencia del gasto público.

El objetivo de la investigación es observar la influencia de variables político/institucionales en la asignación en salud a través de la metodología conocida como "the policy making process» de Spiller, Stein y Tomassi (2008). Se puso especial énfasis en el papel de los actores e instituciones del Órgano Ejecutivo y

1 Uno de los centros de pensamiento más importantes en México enfocado a evaluar el ejercicio del gobierno en México, con la intención de facilitar la toma de decisiones al momento de diseñar políticas públicas. 
Legislativo en cuanto a variables explicativas y en los recursos en salud aprobados por entidad para IMSS, ISSSTE, FASSA y Secretaría de Salud como variable dependiente. Lo anterior, en dos momentos: los últimos tres años del sexenio de Felipe Calderón (PAN) 2010-2012 y los primeros cinco del sexenio de Enrique Peña Nieto (PRI) 2012-2017.

La investigación responde las siguientes preguntas de investigación: ¿El partido político al que pertenece el presidente de la república favorece la asignación de recursos en salud para entidades gobernadas por su mismo partido? ¿El partido político que tiene mayoría en la Cámara de Diputados favorece la asignación de recursos en salud para entidades gobernadas por su mismo partido? Para responderlas, se observaron dos relaciones:

1. La relación entre el partido político en la presidencia y el partido político del gobernador de la entidad.

2. La relación entre el partido político con mayoría en la Cámara de Diputados y el partido político del gobernador de la entidad.

Las relaciones se establecieron a través del cálculo de medias aritméticas de asignación en salud por sexenio para: 1. Entidades gobernadas por el mismo partido que ostenta la presidencia 2. Todas las entidades federativas y 3 . Entidades gobernadas por la oposición. La hipótesis de la investigación es que las entidades gobernadas por el mismo partido al que pertenece el presidente reciben más recursos en salud que las entidades gobernadas por la oposición y que las entidades gobernadas por el mismo partido que tiene mayoría en la Cámara de Diputados reciben más recursos en salud que las entidades gobernadas por la oposición.

Sobre la primera relación, encontramos que la politización de los recursos en salud hacia las entidades federativas depende sobre todo de contar mayoría en la Cámara de Diputados. Durante la presidencia del PAN, por ejemplo, no se observa mayor asignación en salud para las entidades gobernadas por su partido, sino por el contrario, las entidades gobernadas por la oposición recibieron en promedio más recursos. El PAN no tenía mayoría en la Cámara de Diputados. Con el Partido Revolucionario Institucional (PRI), por su parte, se observa una clara politización del gasto y es que durante la presidencia de Peña Nieto había coincidencia partido en la presidencia y mayoría en la Cámara de Diputados. Entre 2013 y 2017 de manera sistemática e ininterrumpida entidades federativas gobernadas por el PRI recibieron una cantidad promedio mayor de recursos que entidades gobernadas por la oposición. A su vez, todos los años en los que el PRI tuvo mayoría en la Cámara de Diputados las entidades gobernadas por el PRI recibieron en promedio más recursos en salud que las entidades gobernadas por la oposición.

\subsection{Presupuesto y política}

En 1940 V. O. Key escribió el clásico libro sobre presupuesto La ausencia de una teoría presupuestal. En él, propone como solución al vacío en el conocimiento, la utilización de la microeconomía como base teórica de las decisiones presupuestales (Le Loup, 2002: ix). Irónicamente, sesenta años después (en el 2000), aún continuamos modelando en busca de una explicación sobre la manera en que deben tomarse las decisiones presupuestales. De acuerdo a Hyde, la falta de coherencia reside en el campo de conocimiento mismo. El presupuesto público es ecléctico y multidimensional. Es en parte político, económico, contable y administrativo (Khan y Hildreth, 2002: x).

1. La parte política de la asignación consiste en la asignación de recursos escasos de una sociedad entre múltiples, competidos y conflictivos intereses.

2. Como documento económico y fiscal es un instrumento básico para evaluar qué tan redistributivo es el ingreso, estimulando crecimiento económico y desarrollo, promoviendo el pleno empleo, combatiendo la inflación y manteniendo estabilidad económica.

3. Como documento contable, provee un tope al gasto gubernamental prohibiendo el desvío de fondos aprobados.

4. Finalmente, como documento administrativo y gerencial, especifica las formas y maneras en que los servicios públicos serán provistos y establece el criterio para su monitoreo, medición y evaluación. Debido a ello, es que resulta difícil que exista una teoría que englobe todos estos elementos en un cuerpo teórico coherente (Khan y Hildreth, 2002: x).

Cuando un único instrumento gubernamental cumple varias funciones a la vez se enfrenta a dilemas y opciones. La figura 1 ilustra los conflictos destacando que cada una de las funciones del presupuesto tiene un norte diferente (Marcel, Guzmán y Sanginés, 2014: 36). Los acuerdos políticos/institucionales entran en conflicto con las variables económicas y gerenciales. 
FIGURA 1. FUNCIONES DEL PRESUPUESTO Y SUS CONFLICTOS

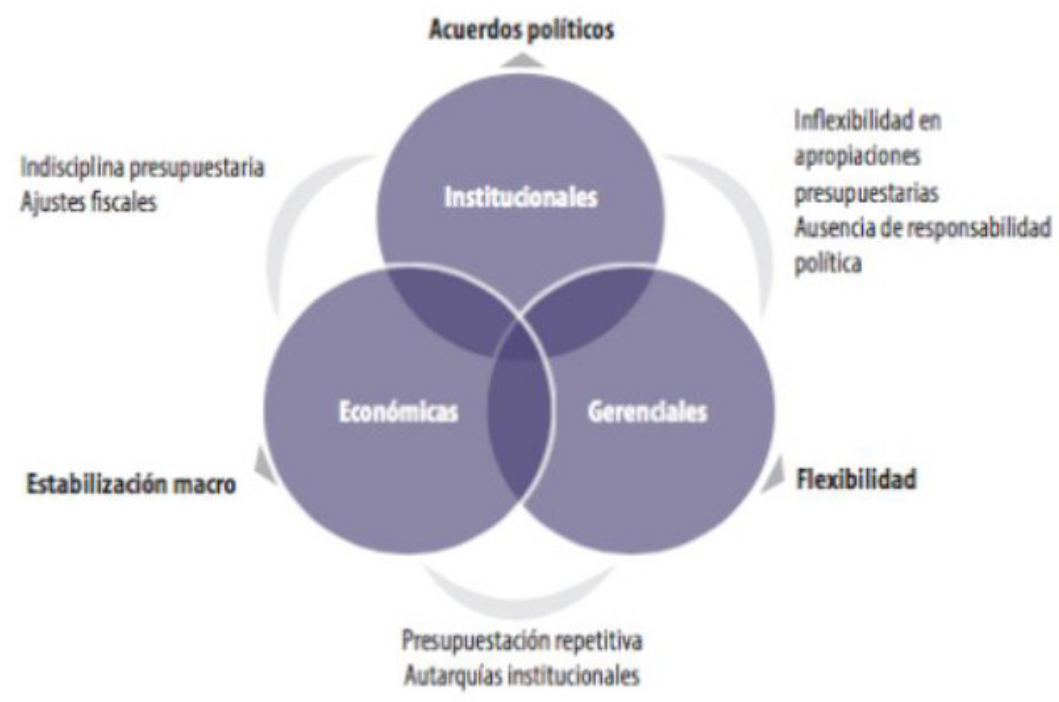

Fuente: Marcel, Guzmán y Sanginés, 2014, pág. 37.

La teoría en materia de presupuesto ha variado mucho en las últimas décadas (Frank, 2007). La teoría de inicio de siglo Xx se basaba primordialmente en la Economía y la Optimización del gasto a través de herramientas como el Presupuesto Base Cero, la Planeación y la Programación. Basadas en este enfoque teórico, las decisiones presupuestales buscaban niveles óptimos de servicios recreativos, reparación de carreteras y número de policías por jurisdicción. La teoría del presupuesto del siglo XXI: Teorías sobre Administración del Desempeño, ponía énfasis en la efectividad del gasto. Basadas en este enfoque teórico, las decisiones presupuestales buscaban por ejemplo, que los niveles de recreación, reparación de carreteras y policías fueran consistentes con los objetivos, las expectativas de la ciudadanía, y el costo y calidad en jurisdicciones similares (Frank, 2007: 228).

Sobre nuestro objeto de estudio, existen infinidad de investigaciones que contemplan variables político/institucionales como explicativas de la decisión presupuestal. Entre ellas, el trabajo de Sørensen (2003), que estudia la política económica de las asignaciones intergubernamentales en Noruega. La investigación de Solé-Ollé y Sorribas-Navarro (2008), que estudia los efectos del alineamiento partidario en la asignación de transferencias intergubernamentales en España. Abuelafia et al. (2009) analizan instituciones presupuestales informales en Argentina. El estudio de la OCDE (2009) ${ }^{2}$ vincula el impacto de las elecciones en el presupuesto. Lema y Streb (2013) estudiaron el alineamiento político y los ciclos presupuestarios y Spiller, Stein y Tommasi (2008), quienes fueron la base analítica de esta investigación, resaltan el proceso político de la decisión como central en la política resultante. En México, como mencionamos, la investigación de México Evalúa (2011) ${ }^{3}$ identifica la politización del gasto en salud como una de las variables que explican su inequidad e inefectividad. Espinosa (2014) analiza mecanismos informales de toma de decisiones, su influencia en las negociaciones presupuestales y la respuesta del gasto de las participaciones federales (Espinosa, 2014: 57). Medrano y M. Smith (2017) analizan la inversión estatal en programas sociales tomando como una de sus tres variables explicativas la ocurrencia de año electoral en el estado.

A pesar de ello, investigaciones que contemplen tres variables: presupuesto en salud, influencia de factores político/institucionales y entidades federativas son escasas tanto a nivel nacional como internacional.

\section{MARCO TEÓRICO: THE POLICY MAKING PROCESS}

En The policy making process Spiller, Stein y Tomassi (2008) sostienen que el problema de las políticas no son las políticas en sí sino el proceso detrás de ellas. El decisor no sólo busca maximizar el bienestar

\footnotetext{
2 Revisiting Political Budget Cycles in Latin America.

3 "10 puntos para entender el gasto en salud en México".
} 
público, sino que trae consigo también intereses particulares. El comportamiento de los actores políticos dependerá de:

- las instituciones políticas (el Congreso, el sistema de partidos, y el judicial)

- las reglas institucionales (reglas electorales y reglas constitucionales)

- las reglas de interacción entre ellos y

- sus intereses particulares (Spiller, Stein y Tomassi, 2008: 2).

En ambientes que facilitan acuerdos políticos, la hechura de políticas será más cooperativa y llevará a políticas más efectivas, estables y flexibles (Spiller, Stein y Tomassi, 2008: 2) y viceversa. Cualquier análisis que busque entender la influencia de variables político-institucionales deberá responder algunas de las siguientes preguntas: 1. ¿Quiénes son los actores principales que participan en el proceso? 2. ¿Qué rol y qué poder tienen? 3. ¿Cuáles son sus preferencias, incentivos y capacidades? 4. ¿Cuáles son sus horizontes en el tiempo?

¿En qué arena interactúan y qué características tiene esta arena? 6. ¿Cuál es la naturaleza del intercambio político y las transacciones que llevan a cabo? (Spiller, Stein y Tomassi, 2008: 14-15).

Con respecto al Órgano Ejecutivo y la figura del presidente, aunque las características personales, ideología y factores culturales e históricos moldean sus decisiones, las instituciones determinan la manera en que son elegidos, y el poder que tienen para decidir tiende a ser igual de importante que sus incentivos y su comportamiento (Scartascini, 2009: 32). En algunos escenarios institucionales, los presidentes se inclinan por el bienestar público y diseñan sus políticas tomando en cuenta los intereses generales de la sociedad. En otros escenarios institucionales, los presidentes tienen incentivos mixtos (políticos y personales) que interfieren con el objetivo de servir el interés público general (Scartascini, 2009: 32). ¿De qué depende?

1. Mientras más poderoso constitucionalmente es un presidente más fácil es tomar decisiones a su conveniencia.

2. Si el presidente tiene amplios poderes legislativos las políticas estarán cerca de las preferencias del órgano ejecutivo (Scartascini, 2009: 33).

3. Se considera que el partido es dominante en un sistema presidencialista cuando el presidente y el legislativo son del mismo partido y se considera que existen dificultades para establecer la agenda cuando el presidente y el legislativo son de partido distinto (Scartascini, 2008: 35).

4. La forma en que fue electo el presidente afecta el grado de apoyo popular y determina si la formación de coaliciones es necesaria. En sistemas electorales en los que el candidato ganador obtuvo un alto porcentaje de votos y cuenta con gran legitimidad la necesidad de formar de coaliciones disminuye.

5. Los incentivos detrás de algunas de las elecciones del ejecutivo se pueden explicar también por la duración en el puesto y temas como la reelección.

En el Órgano Legislativo las reglas electorales y la estructura legislativa son determinantes en las decisiones. El método de elección de los representantes por ejemplo, influye fuertemente en los incentivos de los hacedores de política:

1. Cuando los distritos tienen un solo representante y este se elige por mayoría de votos, los legisladores deciden en función de los intereses de sus representados para garantizar su nominación y reelección.

2. Cuando en un distrito hay varios legisladores y estos se eligen en función de la designación en una lista combinado con un sistema de representación proporcional, las decisiones tienden a desviarse de los intereses de los representados.

3. En sistemas de listas cerradas, la disciplina partidaria es mayor ya que los líderes del partido escogen el orden en la lista. En sistemas de listas abiertas, los candidatos compiten entre ellos y deben por tanto cultivar votos.

4. Si los representantes son seleccionados por legislaturas sub nacionales se vuelven actores muy relevantes en algunos temas, como lo son las transferencias intergubernamentales.

5. En sistemas binomiales se fomenta la estabilidad de las coaliciones.

\subsection{Actores en el proceso de formulación y aprobación del PEF en México}

\subsubsection{El Ejecutivo}

En México el sistema presidencialista actual es muy distinto al que se tuvo previo a la alternancia. Hasta 1997, el presidente en México tenía además de sus facultades constitucionales, poderes tan 
amplios como la decisión de posiciones en la alta burocracia con ratificación asegurada, candidaturas a gobiernos estatales y legislaturas con victoria asegurada, iniciativa de ley con niveles de aprobación superiores al $90 \%$, aprobaciones del presupuesto sin modificaciones e incluso la elección de su sucesor. Los contrapesos que constitucionalmente deberían existir en torno a estos temas, eran prácticamente letra muerta. Durante esos años (1934 a 1997), la administración pública tuvo poca o nula autonomía respecto de la política, dado el largo dominio del PRI en el ejercicio del gobierno (Pardo, 2015: 86). Actualmente, el presidente es mucho menos poderoso. Aunque tiene iniciativa de ley y la propuesta del Presupuesto de Egresos de la Federación sale del ejecutivo, depende de la Cámara de Diputados para su aprobación.

La investigación abarca la presidencia de Felipe Calderón Hinojosa del PAN (2006-2012), segunda presidencia del PAN post alternancia y de Enrique Peña Nieto del PRI (2012-2018), que representa el regreso del PRI después de haber perdido su predominio político en el 2000. Felipe Calderón impulsó una estrategia de renovación política. Sin embargo, sus propuestas de reforma se vieron limitadas por un Congreso en el que el PRI fue un fuerte contrincante (Pardo, 2015: 86-91). Además, el gobierno de Calderón tuvo desde sus inicios cuestionamientos en torno a su legitimidad que condicionaron su actuar los primeros años (Pardo, 2015: 92). Durante su administración se promulgó la Ley Federal de Presupuesto y Responsabilidad Hacendaria (LFPRH) (2006), el Presupuesto Basado en Resultados (PbR), el Sistema de Evaluación de Desempeño (SED) y la coordinación y establecimiento de los mecanismos para la transparencia y rendición de cuentas de los gobiernos estatales y municipales en el ejercicio de los recursos federales como política pública permanente en los niveles de gobierno (Pardo, 2015: 98).

El sexenio de Enrique Peña Nieto (2012-2018) inicia en 2012 con el sorpresivo regreso del PRI a la presidencia después de 12 años de gobiernos del PAN. El discurso de inicio de gobierno planteaba la necesidad de implementar reformas estructurales urgentes que lograron ser acordadas en lo que se conoce como Pacto por México: Reforma energética, educativa, político/electoral, en telecomunicaciones. En 2016 se llevó a cabo la Reforma al Presupuesto, que incluía la metodología para su elaboración conocida como «Presupuesto Base Cero» y que contempla que el presupuesto se elabore «sobre una base cero» lo que obligaría a obtener información sobre desempeño y su relación con el presupuesto (Martínez González, 2015: 66). Cabe destacar que en este sexenio, «las Secretarías más importantes fueron ocupadas por personalidades con amplia trayectoria en el PRI y en muchos casos con larga experiencia en funciones ejecutivas en el gobierno federal o en gobiernos estatales y locales (por ejemplo, en el gabinete había cinco ex-gobernadores), aliados políticos de Peña Nieto y miembros de su equipo más cercano durante su mandato como gobernador del Estado de México» (Olmeda y Armesto, 2013: 262).

\subsubsection{El Legislativo}

Como mencionamos, en México, en el proceso anual de formulación y aprobación del Presupuesto de Egresos de la Federación participan dos grupos de actores: Por parte del Legislativo, la Cámara de Diputados y sus Comisiones correspondientes y del lado del Ejecutivo, el Presidente, la Secretaría de Hacienda y Secretaría de Gobernación (SEGOB). En septiembre del año previo al presupuesto bajo discusión, el presidente envía el Proyecto de Presupuesto de Egresos de la Federación (PPEF) a la Cámara de Diputados, quien tiene hasta noviembre para analizar, discutir y aprobar el mismo.

La Secretaría de Hacienda y Crédito Público es la encargada de formular el PPEF y presentarlo al presidente y junto con SEGOB, es la encargada de la negociación entre la Cámara de Diputados y el Ejecutivo (UNICEF, 2014: 4). La Cámara de Diputados por su parte, es el principal actor en la revisión, negociación y aprobación del Presupuesto de Egresos de la Federación (UNICEF, 2014: 6). Al interior de la Cámara, el grupo responsable de dirigir la discusión y revisión del PPEF es la Comisión de Presupuesto y Cuenta Pública. Esta comisión envía información a las demás comisiones temáticas (por ejemplo, la de Salud) para que éstas, en el ámbito de su competencia, puedan emitir su opinión. Una vez estudiado el Proyecto, la Comisión presenta al Pleno de la Cámara un dictamen con los comentarios, modificaciones o reasignaciones propuestas. El dictamen se discute en el Pleno, quien debe aprobar el Presupuesto de Egresos el día 15 de noviembre como fecha límite, según el artículo 42 fracción $\mathrm{V}$ de la LFPyRH (UNICEF, 2014: 6).

Tomando en consideración estos dos grupos de actores así como los actores que la metodología de «the policy making process» identifica como principales en la toma de decisiones, es que se esta- 
GAPP. Nueva Época - N. ${ }^{0}$ 22, noviembre-abril 2019 - ISSN: 1989-8991 - DOI: 10.24965/gapp.i22.10588 - [Págs. 86-101]

blecieron dos variables explicativas como determinantes de la asignación del presupuesto en salud: el Presidente y la Cámara de Diputados. A continuación se muestra la composición de la Cámara de Diputados en la LXI Legislatura, que inició sus funciones el día 1 de septiembre de 2009 y concluyó el 31 de agosto de 2012.

TABLA 1. COMPOSICIÓN POR PARTIDO DE LA LXI LEGISLATURA 2009-2012

\begin{tabular}{|c|c|c|c|c|c|c|c|c|c|c|c|}
\hline $\begin{array}{c}\text { GRUPO } \\
\text { PARLAMENTARIO }\end{array}$ & $\begin{array}{l}\text { MAYORIA } \\
\text { RELATIVA }\end{array}$ & $\%$ & $1 .^{a}$ & $2 .^{a}$ & $3 .^{a}$ & $4 .^{a}$ & $5 .^{a}$ & $\begin{array}{l}\text { REPRESENTACIÓN } \\
\text { PROPORCIONAL }\end{array}$ & $\%$ & TOTAL & $\%$ \\
\hline PRI & 188 & 62,7 & 11 & 12 & 12 & 8 & 11 & 54 & 27,0 & 242 & 48,4 \\
\hline PAN & 68 & 22,7 & 18 & 18 & 16 & 11 & 11 & 74 & 37,0 & 142 & 28,4 \\
\hline PRD & 33 & 11,0 & 3 & 3 & 6 & 7 & 9 & 28 & 14,0 & 61 & 12,2 \\
\hline PVEM & 4 & 1,3 & 4 & 4 & 2 & 5 & 3 & 18 & 9,0 & 22 & 4,4 \\
\hline PT & 4 & 1,3 & 2 & 1 & 1 & 4 & 2 & 10 & 5,0 & 14 & 2,8 \\
\hline NA & 1 & 0,3 & 2 & 2 & 0 & 2 & 1 & 7 & 3,5 & 8 & 1,6 \\
\hline MC & 0 & 0,0 & 0 & 0 & 2 & 2 & 2 & 6 & 3,0 & 6 & 1,2 \\
\hline SP & 2 & 0,7 & 0 & 0 & 1 & 1 & 1 & 3 & 1,5 & 5 & 1,0 \\
\hline TOTAL & 300 & 100,0 & 40 & 40 & 40 & 40 & 40 & 200 & 100,0 & 500 & 100,0 \\
\hline
\end{tabular}

Fuente: Álbum de Diputados Federales 2009-2012.

En la LXI Legislatura, el PRI obtuvo el $48,4 \%$ del total (242 diputados). Si a esto se suma los diputados del PVEM y NA con quien el PRI formó alianza en ese periodo podemos decir que este partido contaba en la LXI Legislatura con 272 votos favorables. Es decir, mayoría absoluta en coalición en las asignaciones presupuestales para el 2010 y 2011. En la XII Legislatura (2012-2015), el PRI obtuvo un total de 214 diputados. Nuevamente, si se suma a los legisladores del PVEM y de NA tenemos un total de 251 legisladores favorables al PRI, alcanzando apenas la mayoría absoluta. La LXII Legislatura fue la base para el análisis de la relación entre mayoría (PRI, PVEM y NA) y asignación en salud a las entidades en 2012, 2013, 2014 y 2015.

TABLA 2. COMPOSICIÓN POR PARTIDO DE LA LXII LEGISLATURA 2012-2015

\begin{tabular}{|c|c|c|c|c|c|c|c|c|c|c|c|}
\hline $\begin{array}{c}\text { GRUPO } \\
\text { PARLAMENTARIO }\end{array}$ & $\begin{array}{l}\text { MAYORIA } \\
\text { RELATIVA }\end{array}$ & $\%$ & $1 .^{a}$ & $2 .^{a}$ & $3 .^{a}$ & $4 .^{a}$ & $5 .^{a}$ & $\begin{array}{l}\text { REPRESENTACIÓN } \\
\text { PROPORCIONAL }\end{array}$ & $\%$ & TOTAL & $\%$ \\
\hline PRI & 165 & 55,0 & 11 & 10 & 10 & 7 & 11 & 49 & 24,5 & 214 & 42,8 \\
\hline PAN & 51 & 17,0 & 14 & 18 & 11 & 9 & 10 & 62 & 31,0 & 113 & 22,6 \\
\hline PRD & 57 & 19,0 & 5 & 5 & 9 & 12 & 11 & 42 & 21,0 & 99 & 19,8 \\
\hline PVEM & 12 & 4,0 & 2 & 3 & 4 & 3 & 3 & 15 & 7,5 & 27 & 5,4 \\
\hline MC & 5 & 1,7 & 3 & 0 & 1 & 2 & 1 & 7 & 3,5 & 12 & 2,4 \\
\hline PT & 2 & 0,7 & 1 & 2 & 2 & 2 & 2 & 9 & 4,5 & 11 & 2,2 \\
\hline NA & 0 & 0,0 & 2 & 2 & 2 & 2 & 2 & 10 & 5,0 & 10 & 2,0 \\
\hline MORENA & 6 & 2,0 & 2 & 0 & 1 & 3 & 0 & 6 & 3,0 & 12 & 2,4 \\
\hline SIN PARTIDO & 2 & 0,7 & & & & & & 0 & & 2 & 0,4 \\
\hline TOTAL & 300 & 100,0 & 40 & 40 & 40 & 40 & 40 & 200 & 100,0 & 500 & 100,0 \\
\hline
\end{tabular}

Fuente: Álbum de Diputados Federales 2012-2015. 
GAPP. Nueva Época - N. ${ }^{0}$ 22, noviembre-abril 2019 - ISSN: 1989-8991 - DOI: 10.24965/gapp.i22.10588 - [Págs. 86-101]

Consideraciones políticas de la asignación presupuestal en salud en México 2010-2017

Gabriela Tapia Téllez

Finalmente, la LXIII Legislatura inició funciones el día 1 de septiembre de 2015 para concluir el 31 de agosto de 2018 con la siguiente composición:

TABLA 3. COMPOSICIÓN DE LA LXIII LEGISLATURA POR PARTIDO

\begin{tabular}{|c|c|c|c|c|c|c|c|c|c|c|c|}
\hline $\begin{array}{c}\text { GRUPO } \\
\text { PARLAMENTARIO }\end{array}$ & $\begin{array}{l}\text { MAYORIA } \\
\text { RELATIVA }\end{array}$ & $\%$ & $1 .^{a}$ & $2 .^{a}$ & $3 .^{a}$ & $4 .^{a}$ & $5 .^{a}$ & $\begin{array}{l}\text { REPRESENTACIÓN } \\
\text { PROPORCIONAL }\end{array}$ & $\%$ & TOTAL & $\%$ \\
\hline PRI & 158 & 77,1 & 10 & 10 & 10 & 6 & 11 & 47 & 22,9 & 205 & 41,0 \\
\hline PAN & 56 & 51,4 & 13 & 15 & 8 & 8 & 9 & 53 & 48,6 & 109 & 21,8 \\
\hline PRD & 29 & 53,7 & 2 & 3 & 6 & 7 & 7 & 25 & 46,3 & 54 & 10,8 \\
\hline MORENA & 22 & 46,8 & 3 & 2 & 5 & 10 & 5 & 25 & 53,2 & 47 & 9,4 \\
\hline PVEM & 22 & 55,0 & 2 & 4 & 7 & 3 & 2 & 18 & 45,0 & 40 & 8,0 \\
\hline MC & 10 & 50,0 & 4 & 2 & 1 & 2 & 1 & 10 & 50,0 & 20 & 4,0 \\
\hline NA & 1 & 8,3 & 2 & 2 & 2 & 3 & 2 & 11 & 91,7 & 12 & 2,4 \\
\hline PES & 0 & 0,0 & 4 & 1 & 1 & 1 & 2 & 9 & 100,0 & 9 & 1,8 \\
\hline SP & 1 & 33,3 & 0 & 1 & 0 & 0 & 1 & 2 & 66,7 & 3 & 0,6 \\
\hline IND & 1 & 50,0 & 0 & 0 & 0 & 0 & 0 & 0 & 0,0 & 1 & 0,2 \\
\hline TOTAL & 300 & 60 & 40 & 40 & 40 & 40 & 40 & 200 & 40 & 500 & 100 \\
\hline
\end{tabular}

Fuente: Cámara de Diputados 2017 (Datos al 30-10-2017).

EI PRI obtuvo 205 legisladores. Si a esos diputados agregamos los 40 del PVEM y los 12 de Nueva Alianza el PRI contaba con un total de 257 votos favorables. Es decir, mayoría absoluta holgada en coalición. La LXIII Legislatura fue la base para el análisis de la relación entre esta mayoría en la Cámara y la asignación en salud a las entidades en 2016 y 2017.

Es importante recalcar que esta mayoría se hace presente de diversas formas en el proceso de asignación presupuestal. Por un lado, con los votos a favor o en contra del PPEF y el PEF, pero también al interior de las distintas Comisiones de la Cámara de Diputados en las que se discute el presupuesto. De acuerdo al art. 43 de la Ley Orgánica del Congreso de la Unión, en la integración de las Comisiones se toma en cuenta la pluralidad representada en la Cámara así como el criterio de proporcionalidad del Pleno (UNICEF, 8). Por ejemplo, en la LXI Legislatura, en la Comisión de Presupuesto y Cuenta Pública el $45 \%$ de los miembros eran del PRI y en la Comisión de Salud, el $47 \%$ de los miembros eran del PRI. A su vez, cada Comisión tiene una Mesa Directiva, la cual es integrada por un presidente y varios secretarios. EI PRI tuvo predominio en la composición de todas las mesas directivas, entre ellas, la de Presupuesto y Cuenta Pública, donde el $43 \%$ de los miembros eran de su partido y la de Salud, donde contaba con el $50 \%$.

Durante el periodo bajo análisis (2010-2017), si la hipótesis se confirma, debido a que el PRI cuenta con mayoría en la Cámara de Diputados todos los años, las entidades federativas gobernadas por el PRI deberían recibir más recursos en salud.

\section{METODOLOGÍA}

Con base en el marco teórico «the policy making process» y el proceso establecido de manera institucional para la elaboración y formulación del presupuesto en México presentado en las secciones anteriores se seleccionaron las siguientes variables para análisis: 
- Las variables independientes o explicativas X1 y X2.

X1: Coincidencia de partido político en la presidencia y partido político que gobierna en la entidad.

X2: Coincidencia de partido político con mayoría en la Cámara de Diputados y partido político del gobernador en la entidad.

- La variable dependiente Y.

Y: Monto total anual por persona que se asigna a la salud en cada entidad (incluye: gasto en obra de la SS + gasto en equipo de la SS + Inversión federal IMSS + Inversión federal ISSSTE+ FASSA, RAMO33).

Se sumó el recurso asignado en el Presupuesto de Egresos de la Federación (PEF) 2010-2017 para el IMSS, ISSSTE y FASSA con el gasto identificado en los Planes Maestros de Infraestructura (PMI) de la Secretaría de Salud 2010-2017.

1. El Fondo de Aportaciones para los Servicios de Salud (FASSA) del Ramo 33 asigna recursos a las entidades Federativas y municipios a partir de fórmulas y procedimientos establecidos en la Ley de Coordinación Fiscal (LCF). Las transferencias de los fondos del Ramo 33 se aprueban por la Cámara de Diputados en el ejercicio fiscal aplicable y tienen como objetivo fortalecer los Servicios Estatales de Salud para que respondan con mayor eficacia y eficiencia las necesidades de salud de la población sin seguridad social. Actualmente los recursos otorgados a FASSA representan el $16 \%$ del presupuesto total del sector salud (Belisario Domínguez, 2018: 1).

2. Los programas y proyectos de inversión del Instituto Mexicano del Seguro Social (IMSS) y el Instituto de Seguridad y Servicios Sociales de los Trabajadores del Estado (ISSSTE). Estos programas son aprobados por la Cámara de Diputados en el ejercicio fiscal aplicable y representan alrededor del $7 \%$ del gasto de inversión en salud (Belisario Domínguez, 2018: 5). Las dependencias y entidades de la administración pública federal detectan necesidades de inversión, planean y analizan las alternativas que resuelven esas necesidades. La Cámara de Diputados selecciona las alternativas con mayor beneficio social y que cumplen con la normativa en materia de inversión: de impacto regional, que permitan alcanzar las metas contenidas en el Plan Nacional de Infraestructura y en el Plan Nacional de Desarrollo (SHYCP, 2012: 2-3)

3. Los Planes Maestros de Infraestructura de Secretaría de Salud: Rubro Infraestructura y Equipo por entidad. Es el instrumento rector para la planeación, desarrollo, reordenamiento de la infraestructura y el equipamiento médico de la Secretaría de Salud y los Servicios Estatales de Salud, con el fin de racionalizar y priorizar los recursos para la inversión pública y garantizar la operación sustentable.

La relación entre variables $\mathrm{X} 1$ y $\mathrm{X} 2$ con variable $\mathrm{Y}$ se observó a través de:

1. Promedios de asignatura en salud de los últimos años del sexenio de Felipe Calderón 2010-2012 en entidades en las que hay coincidencia presidente (PAN) y gobernador (PAN) versus promedios de asignatura en entidades en las que gobernaba la oposición.

2. Promedios de asignatura 2013-2017 en el sexenio de Enrique Peña Nieto en entidades en las que hay coincidencia presidente (PRI) y gobernador (PRI) versus promedios de asignatura en entidades en las que gobernaba la oposición.

3. Promedios de asignatura 2010-2017 en entidades en las que hay coincidencia mayoría en la cámara (PRI/PVEM) y gobernador (PRI, PVEM) del mismo partido versus promedios de asignatura en entidades donde el gobernador es de oposición.

Las hipótesis:

1. Las entidades gobernadas por el mismo partido que ostenta la presidencia reciben más recursos en salud en el periodo 2010-2017.

2. Las entidades gobernadas por partidos de oposición al que ostenta la presidencia reciben menos recursos en salud para el periodo 2010-2017.

3. Las entidades gobernadas por el mismo partido que tiene mayoría en la Cámara de Diputados reciben más recursos en salud en el periodo 2010-2017. 
4. Las entidades gobernadas por un partido de oposición al que tiene mayoría en la Cámara de Diputados reciben menos recursos en salud en el periodo 2010-2017.

\subsection{La variable Partido del Presidente como explicativa a la asignación de recursos en salud a las entidades federativas}

De acuerdo con Scarstacini (2008), aunque las características personales, ideología y factores culturales moldean las decisiones presidenciales, las instituciones determinan la manera en que son elegidos y el poder que tienen para decidir tiende a ser igual de importante que sus incentivos y comportamiento. En México, aunque el presidente hasta 1997 solía ser sumamente poderoso y sus deseos eran prácticamente órdenes, ese poder era resultado de cuatro características que actualmente no están presentes en el sistema político. 1. Un sistema presidencialista basado en la constitución 2. Gobierno unificado 3. Disciplina al interior del partido 4. Presidente como líder del partido (Weldon, 1997). La investigación deja ver que el partido al que pertenece el presidente por sí sólo no garantiza mayores asignaciones presupuestales en salud a entidades federativas del mismo partido político. Sin embargo, cuando el presidente cuenta con mayoría en la Cámara de Diputados, sí se observa una politización del gasto en salud hacia las entidades federativas del mismo partido.

\subsubsection{La No politización de las asignaciones en salud durante el sexenio de Felipe Calderón}

Durante el sexenio de Felipe Calderón (PAN), el PAN no tenía mayoría en la Cámara de Diputados. Como se observa en las siguientes gráficas. Los últimos tres años de su sexenio no reflejan una politización de la asignación de los recursos en salud a favor del PAN ya que en México, la aprobación del presupuesto es un trabajo conjunto del Ejecutivo con el Legislativo. Las mayores asignaciones percápita por entidad entre 2010 y 2012 son para Campeche, Nayarit, Aguascalientes y Sinaloa. De estas cuatro entidades, solamente Sinaloa estaba gobernada por el PAN en este periodo. Campeche, Nayarit y Aguascalientes estaban gobernadas por partidos de oposición.

FIGURA 2. ASIGNACIÓN PERCÁPITA PROMEDIO EN SALUD POR ENTIDAD 2010-2012

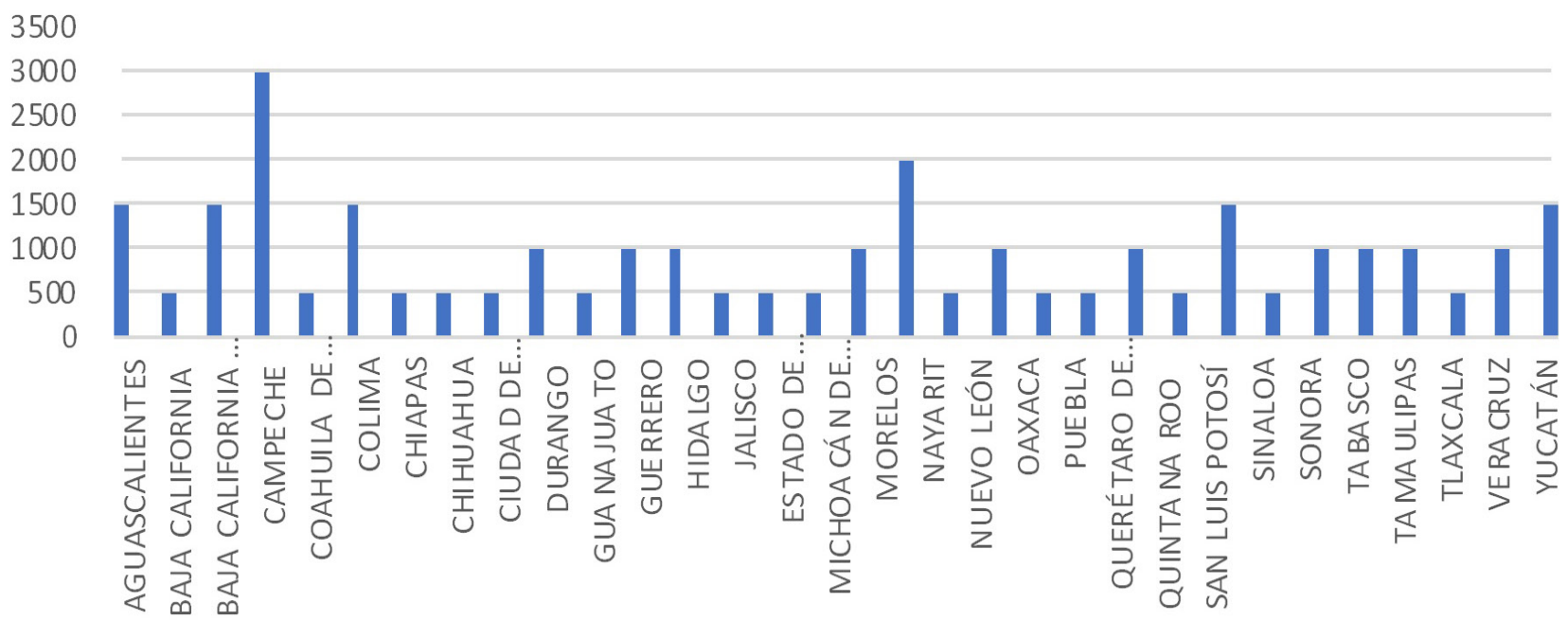

Fuente: Elaboración propia con base en PEF 2010-2017 y PMI Secretaría de Salud 2010-2017.

La siguiente gráfica nos confirma la ausencia de relación entre partido político en la presidencia y cantidad de recursos que bajan a la entidad. Incluso, se observa lo contrario. La barra azul muestra el promedio del monto total anual que recibe en salud una persona que vive en una entidad gobernada por el PAN durante 2010-2012. Dado que el presidente en ese momento era del PAN, uno esperaría observar que la barra azul fuera más alta que la roja, que representa el promedio que recibe una persona en una entidad goberna- 
da por la oposición. Sin embargo, se observa lo contrario. Las entidades gobernadas por partidos distintos al que ocupa la presidencia (PAN Presidencia-Oposición en la entidad) reciben un promedio total anual por persona más alto los tres años bajo análisis que las entidades gobernadas por el mismo partido que el de la presidencia (PAN Presidencia-PAN en la entidad).

Figura 3. PROMEdio del MONTO TOTAL ANUAL POR PERSONA QUE SE ASIGNAN A LA SALUd SEGÚN GRUPOS DE ENTIDADES, 2010-2012

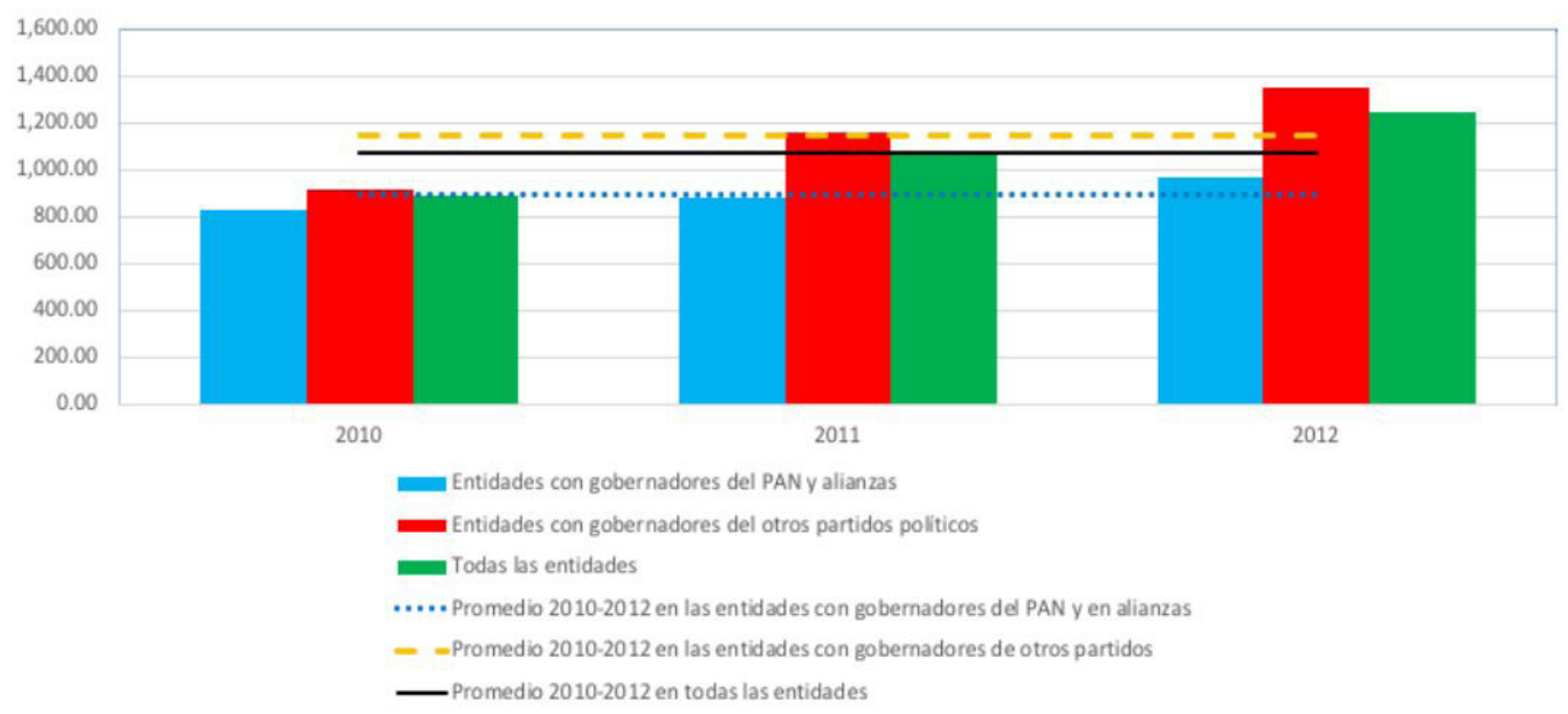

Fuente: Elaboración propia con base en PEF 2010-2017, PMI Secretaría de Salud 2010-2017 y Álbum de Diputados LXI, LXII y LXIII Legislatura.

El no contar con mayoría en la Cámara de Diputados es uno de los factores con más peso para explicar estas asignaciones. Sin embargo, no debemos dejar de lado que también puede explicarse por factores que Scarstascini (2008) identifica sobre las presidencias en las que el candidato ganador obtiene un bajo porcentaje de votos al llegar a la presidencia y poca legitimidad. En estos contextos, la necesidad de formar coaliciones aumenta y es poco probable que las preferencias del órgano ejecutivo se reflejen en las políticas. Calderón llega a la presidencia con bajos niveles de aprobación, con cuestionamientos sobre su victoria y conforme fue avanzando el sexenio, con un desgaste político aún mayor por los problemas de violencia que se desataron en el país. El ánimo de calmar la tensión inicialmente, con la ausencia de poder político que fue acrecentándose y una Cámara de Diputados de Oposición probablemente lo obligó a conceder asignar más recursos a entidades de oposición. Esto, junto con la agenda de renovación política que abanderaba el PAN en esos años se reflejó en asignaciones en salud más grandes para estos partidos muy probablemente con objetivos conciliatorios y de pacificación.

\subsubsection{La politización de las asignaciones en el sexenio de Enrique Peña Nieto}

A diferencia de Calderón, Enrique Peña Nieto asume la presidencia con niveles de legitimidad muy por encima de los que tuvo Calderón y además con mayoría en la Cámara de Diputados. Estas variables permiten explicar las decisiones del PRI durante ese sexenio, que claramente favorecen a entidades federativas del mismo partido en detrimento de los habitantes que viven en entidades gobernadas por la oposición.

En la siguiente gráfica se puede apreciar que en los primeros cinco años del sexenio de EPN (20132017), las cuatro entidades con mayor asignación percápita (Campeche, Aguascalientes, Nayarit y Colima) fueron también entidades que en ese periodo estaban gobernadas por el PRI. 
FIGURA 4. ASIGNACIÓN PERCÁPITA PROMEDIO EN SALUd 2013-2017 POR ENTIDAD



Fuente: Elaboración propia con base en PEF 2010-2017, PMI Secretaría de Salud 2010-2017.

FIGURA 5. PROMEDIO DEL MONTO ANUAL POR PERSONA QUE SE ASIGNAN A LA SALUd SEGÚN GRUPOS DE ENTIDADES 2013-2017

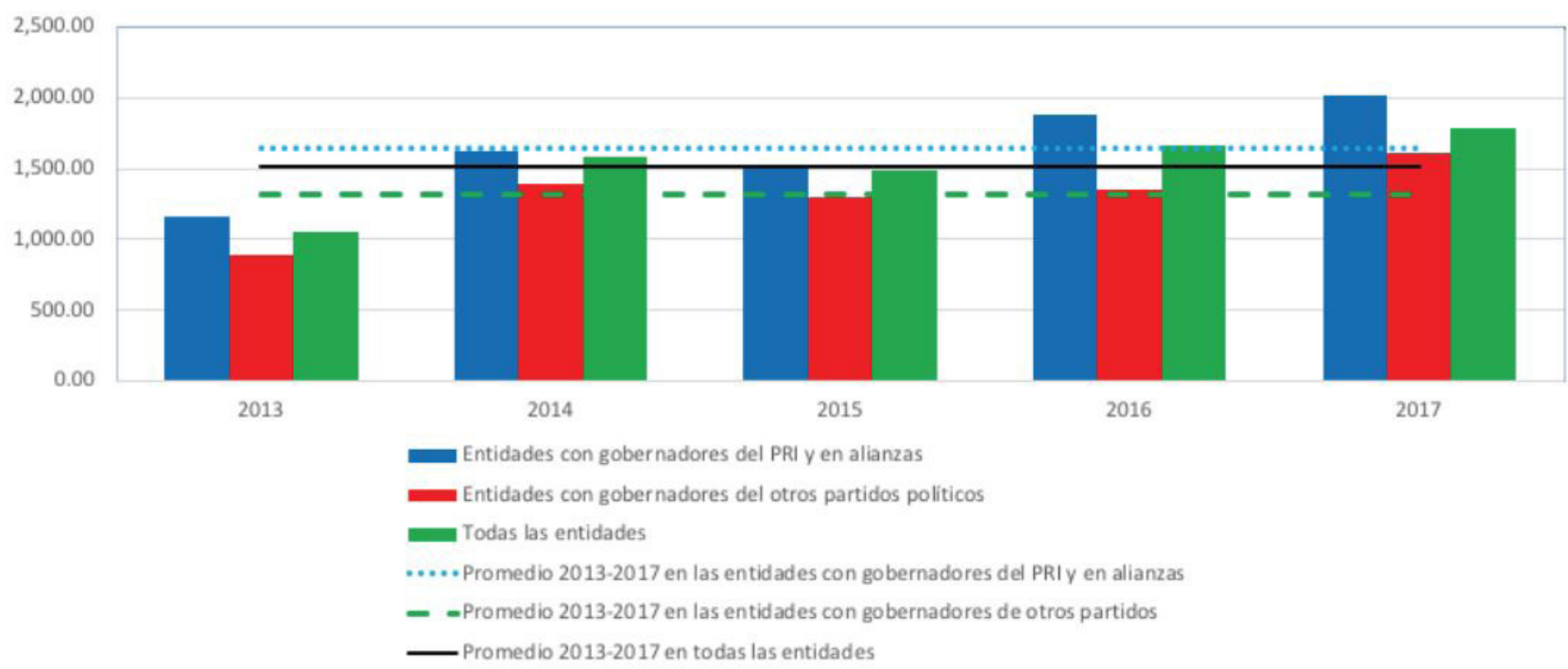

Fuente: Elaboración propia con base en PEF 2010-2017, PMI Secretaría de Salud 2010-2017 y Album de Diputados LXI, LXII y LXIII Legislatura. 
La gráfica anterior nos brinda más certeza sobre el periodo 2013-2017. Como se puede ver, las entidades gobernadas por el PRI reciben todos los años un mayor promedio de recursos en salud por persona que las entidades gobernadas por la oposición. La columna azul muestra el promedio del monto anual por persona que se asigna a salud para entidades gobernadas por el PRI. La columna roja representa el promedio del monto anual por persona que se asigna a salud a entidades gobernadas por la oposición. En los cinco años bajo análisis (2013-2017) las entidades gobernadas por el PRI tuvieron siempre un promedio anual de asignación en salud por persona más alto que las entidades gobernadas por la oposición.

Debemos tener en cuenta que el partido al que pertenece el presidente no es la única variable que explica estas asignaciones. De ser así, en el sexenio de Calderón hubiéramos observado mayores asignaciones para entidades federativas panistas. De acuerdo con las entrevistas de la investigación de Espinosa (2014) «contar con una relación directa con el presidente y miembros estratégicos del gabinete (en particular, secretarios y subsecretarios de Estado) aún tiene una importancia considerable cuando se negocian apoyos financieros adicionales a los obtenidos por medio de los canales presupuestales formales» (Espinosa, 2014: 69). Sin embargo, como veremos, la mayoría en la Cámara de Diputados, parece explicar en mayor medida la capacidad de dirigir recursos hacia entidades federativas del mismo partido político durante la elaboración del PEF.

\subsection{La variable Mayoría en la Cámara de Diputados como explicativa a la asignación de recursos en salud a las entidades federativas}

La variable de mayoría en la Cámara de Diputados parece ser un mejor predictor de los recursos en salud que recibirán las entidades federativas que la variable partido político del presidente. Durante los ocho años bajo análisis el PRI gozó de mayoría en la Cámara de Diputados y las entidades federativas gobernadas por el PRI recibieron sistemática e ininterrumpidamente más recursos en salud.

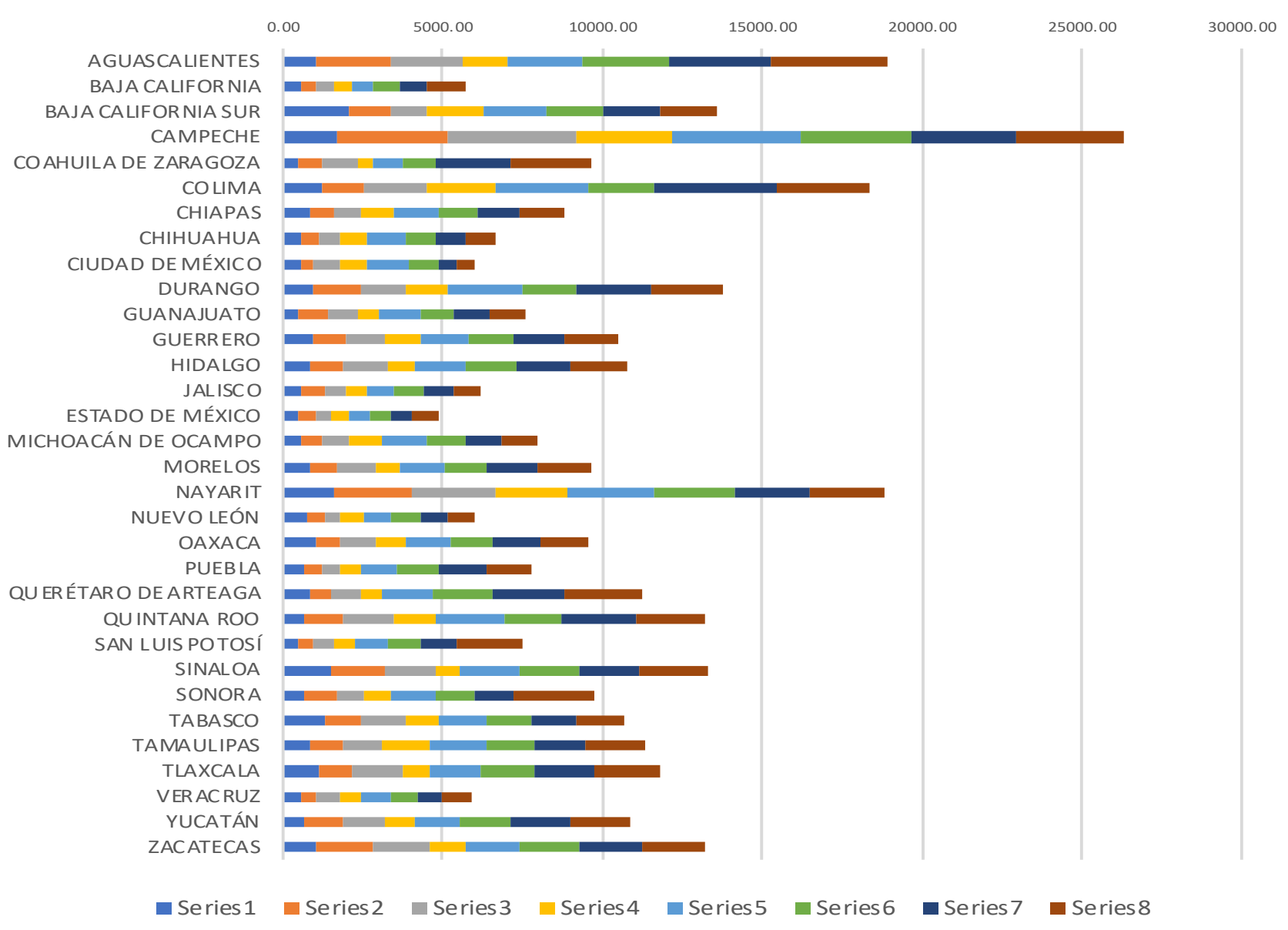

Fuente: Elaboración propia con base en PEF 2010-2017, PMI Secretaría de Salud 2010-2017. 
La anterior gráfica muestra el monto total anual asignado por persona en salud por entidad federativa en el periodo 2010-2017. Como se puede observar Campeche, Aguascalientes, Colima y Nayarit han sido las entidades cuya población ha sido asignada la mayor cantidad de recursos en salud percápita en los últimos siete años. Las cuatro entidades estaban gobernadas por el PRI. Es decir, existe coincidencia (mayoría del PRI en la Cámara de Diputados y PRI en gubernatura).

En la siguiente gráfica se observa también que los ocho años en los que el PRI tuvo mayoría en la Cámara de Diputados la asignación en salud promedio para las entidades gobernadas por el PRI (columna azul) siempre fue mayor que en las entidades gobernadas por la oposición (columna roja) y mayor siempre también que el promedio de asignación anual de todas las entidades juntas (columna verde).

\section{FIGURA 7. PROMEDIO DEL MONTO TOTAL ANUAL POR PERSONA QUE SE ASIGNAN A LA SALUD SEGÚN GRUPOS DE ENTIDADES 2010-2017}



Fuente: elaboración propia con base en PEF 2010-2017, PMI Secretaría de Salud 2010-2017 y Album de Diputados LXI, LXII y LXIII Legislatura.

Es decir, se observa una clara politización del gasto en salud que se explica por la variable de mayoría del PRI en la Cámara de Diputados. De acuerdo a las entrevistas de Espinosa (2014), «la capacidad de las autoridades estatales para atraer fondos federales adicionales aumenta cuando el gobernador tiene aliados políticos cercanos en comisiones estratégicas en la Cámara de Diputados (v. gr., las comisiones de presupuesto, al interior de las cuales se definen las asignaciones de gastos federales).... aunque los gobernadores no participan de manera formal en la integración de las comisiones legislativas, lograr que su partido político obtenga resultados electorales favorables en su estado natal puede otorgarles el poder para negociar posiciones para sus aliados con los líderes de su partido. El orden institucional existente y la enorme dependencia que tienen los estados de las participaciones federales, incentiva a los gobernadores a fungir como agentes electorales. las necesidades de gasto público y para entregar resultados tangibles en el ámbito estatal» (Espinosa, 2014: 70).

\section{CONCLUSIONES}

La investigación muestra que los pesos y contrapesos establecidos en la Constitución para la formulación del presupuesto en México no lo exentan de la influencia de factores políticos en su asignación. La presencia de variables como mayoría en la Cámara de Diputados, altos niveles de legitimidad y coincidencia con partido que gobierna en la entidad son determinantes importantes de que los recursos se politicen. Por el contrario, también pudimos constatar que el presidente por sí solo no parece tener poderes que le permitan tomar decisiones cercanas a sus preferencias. Cuando el presidente no cuenta con mayoría en la Cámara 
de Diputados, como sucedió durante la administración del PAN con Calderón (2006-2012) y encima existen problemas de legitimidad, el presidente se ve obligado a negociar con la oposición y tomar decisiones que no necesariamente lo favorecen a él ni a su partido. Evidencia de ello es que durante este sexenio, las entidades federativas panistas, no recibieron en promedio más recursos percápita para salud, sino por el contrario, las entidades gobernadas por la oposición recibieron mayores asignaciones.

En el caso del sexenio del PRI, donde existe coincidencia Mayoría en la Cámara de Diputados, gobierno con altos niveles de aprobación y entidades gobernadas por el mismo partido, se observa una fuerte politización de los recursos en salud. Entre 2013 y 2017 todos los años sin excepción se observan dos situaciones: una coincidencia entre partido del presidente (PRI) y una mayor cantidad promedio de recursos en salud para entidades federativas del PRI y dos, una coincidencia entre mayoría del PRI en la Cámara de Diputados y una mayor cantidad promedio de recursos en salud para entidades federativas del mismo partido.

EI PRI se ha caracterizado históricamente por una fuerte disciplina partidaria. En años previos a la alternancia, los premios a los gobernadores más leales y disciplinados se traducían en mayores asignaciones presupuestales. Actualmente, en un contexto de alternancia política, los «premios» y «castigos» parecieran dirigirse a gobernadores del mismo partido político. Si a este incentivo le sumas la enorme dependencia financiera que tienen las entidades federativas de los recursos federales, es fácil entender que los incentivos para que los gobiernos estatales recurran a mecanismos de negociación que no están contemplados, de manera explícita, en las leyes presupuestales sean tan altos. Lo anterior es grave de muchas formas, pero sobre todo porque implica la apropiación de recursos públicos con intereses partidistas en un contexto de gran inequidad en materia de salud en las entidades federativas del país.

Actualmente, aunque tuvimos alternancia política y la izquierda (Morena) llega por primera ver a ocupar la presidencia de México nuevamente están presentes variables que podrían politizar el presupuesto en salud: gobierno unificado, altos niveles de legitimidad y cinco entidades gobernadas por este mismo partido político. Esperemos que la distribución de recursos este sexenio refleje una verdadera vocación de redistribución y los recursos se dirijan a las entidades con mayores necesidades en salud.

\section{REFERENCIAS BIBLIOGRÁFICAS}

CÁMARA DE DIPUTADOS (2012): Álbum de Diputados Federales 2009-2012. Legislatura LXI (08 de agosto de 2012). URL: http://sitllxi.diputados.gob.mx/album_dips.pdf [accesado: 08-11-2017].

CÁMARA DE DIPUTADOS (2015): Álbum de Diputados Federales 2012-2015. Legislatura LXII (14 de agosto de 2015). URL: http://sitl/xii.diputados.gob.mx/album_foto_tc.pdf [accesado: 08-11-2017].

CÁMARA DE DIPUTADOS (2017): LXIII Legislatura. URL: http://sitllxiii.diputados.gob.mx/info_diputados.php [accesado: 08-11-2017].

CONEVAL (2018): Estudio diagnóstico del derecho a la salud 2018. Ciudad de México: CONEVAL. URL: https://www. coneval.org.mx/Evaluacion/IEPSM/Documents/Derechos_Sociales/Diag_derecho_Salud_2018.pdf.

ESPINOSA, S. (2014): "Mecanismos informales de negociación presupuestal y el impacto de las participaciones en el gasto de los estados. El caso de Baja California, México", en Gestión y Política Pública, vol. XXIII, núm. 1, págs. 47-78.

INSTITUTO BELISARIO DOMÍNGUEZ (2018): Recursos destinados al sector salud en el Presupuesto de Egresos de la Federación 2018. México: Senado de la República, Dirección General de Finanzas. Serie: Seguimiento al Ciclo Presupuestario. LXIII Legislatura.

KHAN, A. y HILDRETH, W. B. (2002): "Preface”, en KHAN, A. y HILDRETH, W. B. (ed.): Budget Theory in the Public Sector. Connecticut, London: Quorum books.

LE LOUP, L. T. y MORELAND, W. B. (1978), "Agency strategies and executive review: The Hidden politics of budgeting”, en Public Administration Review, vol. 38, núm. 3. DOI: https://doi.org/10.4067/10.2307/975675.

THOMAS, L. P. (2002): “The Separation of Powers Principle and Budget Decision”, en KHAN, A. y HILDRETH, W. B. (ed.): Budget Theory in the Public Sector. Connecticut, London: Quorum books.

MAJONE, G. (2008): Evidencia, argumentación y persuasión en la formulación de políticas. México: Fondo de Cultura Económica.

MARCEL, M., GUZMÁN, M. y SANGINÉS, M. (2014): Presupuestos para el desarrollo en América Latina. Washington: BID.

MARTíNEZ GONZÁLEZ, J. G. (2015): "Reforma al Presupuesto Federal", en El cotidiano, núm. 192, págs. 63-68.

MEDRANO, A. y SMITH, H. J. M. (2017): "Inversión estatal en programas sociales a tres décadas de la descentralización y la reforma social en México", en Gestión y Política Pública, vol. 26, núm. especial, págs. 157-189. 
MÉXICO EVALÚA (2011): "10 puntos para entender el gasto en salud en México: En el Marco de la Cobertura Universal”. URL: https://www.mexicoevalua.org/2011/01/01/10-puntos-para-entender-el-gasto-en-salud-en-mexicoen-el-marco-de-la-cobertura-universal/.

MINISTERIO DE SALUD (31 de marzo 2016): "Informe de brechas sobre personal de salud por servicio de salud". Santiago de Chile. URL: http://web.minsal.cl/wp-content/uploads/2015/08/Informe-Brechas-RHS-en-SectorP\%C3\%BAblico_Marzo2016.pdf.

OLMEDA, J. y ARMESTO, M. A. (2013): "México: el regreso del PRI a la presidencia", en Revista de Ciencia Política, vol. 33, núm. 1, pág. 247-267. DOI: https://doi.org/10.4067/s0718-090x2013000100012.

PARDO, M. ${ }^{\text {a C }}$ (2015): "La modernización administrativa del gobierno de Felipe Calderón: Entre la austeridad y la reforma", en Foro Internacional, vol. LV, núm. 219, págs. 83-115. DOI: https://doi.org/10.24201/fi.v55i1.2263.

SCARTASCINI, C. (2008): "Who's Who in the PMP: An Overview of Actors, Incentives and the Roles They Play", en STEIN, E. y TOMMASI, M. (eds.): Policymaking in Latin America: How Politics Shapes Policies, págs. 29-68. Washington DC: Interamerican Development Bank, David Rockefeller Center for Latin American Studies. DOI: https://doi.org/10.2139/ssrn.1719386.

SECRETARÍA DE HACIENDA Y CRÉDITO PÚBLICO (2012): "Registro en cartera de programas y proyectos de inversión". Subsecretaría de Egresos, Unidad de Inversiones. URL: http://www.cmic.org.mx/comisiones/sectoriales/ infraestructurahidraulica/programas\%20SHCP/RegistroenCarteraenProgramasyProyectosdelnversion.pdf.

SOLÉ-OLLÉ, A. y SORRIBAS-NAVARRO, P. (2008): "The Effects of Partisan Alignment on the Allocation of Intergovernmental Transfers: Differences-indifferences Estimates for Spain”, en Journal of Public Economics, vol. 92, núm. 12, págs. 2.302-2.319. DOI: https://doi.org/10.1016/j.jpubeco.2007.06.014.

SØRENSEN, R. J. (2003): "The Political Economy of Intergovernmental Grants: The Norwegian Case”, en European Journal of Political Research, vol. 42, núm. 2, págs. 163-195. DOI: https://doi.org/10.1111/1475-6765.00079.

SPILLER, P., STEIN, E. y TOMMASI, M. (2008): "Political Institutions, Policymaking, and Policy: An Introduction", en STEIN, E. y TOMMASI, M. (eds.): Policymaking in Latin America: How Politics Shapes Policies, págs. 1-28. Washington DC: Interamerican Development Bank, David Rockefeller Center for Latin American Studies.

UNICEF (2014): El proceso presupuestario en México y su contexto político.

WELDON, J. (1997): "Las fuentes políticas del presidencialismo en México", en MAINWARING, S. y SOBERING, M. (eds.): Presidencialismo y democracia en América Latina. Buenos Aires: Paidós. 